\title{
Pilot study on the utility and acceptability of video animation as a delivery method for educational materials for families of patients with congenital hyperinsulinism in infancy
}

\author{
E Rodgers ${ }^{1}$, L Rigby², MJ Dunne ${ }^{1}$, I Banerjee ${ }^{2}$ and KE Cosgrove ${ }^{1}$
}

${ }^{1}$ Faculty of Life Sciences University of Manchester, UK; ²Paediatric Endocrinology Department, Royal Manchester Children's Hospital, UK.

Introduction

To prevent neurological damage caused by congenital hyperinsulinism $(\mathrm{CHI})$ hypoglycaemia must be avoided and treated promptly. Education of parents, carers and families (PCFs) of patients with $\mathrm{CHI}$ about the causes and consequences of $\mathrm{CHI}$ may help to reduce severity of hypoglycaemia due to earlier correction of blood glucose levels. PCFs currently learn about $\mathrm{CHI}$ via interaction with health care providers (HCPs) and via leaflets available from the hospital, although "unofficial" channels of information are readily available via the internet and social media. Provision of high quality digital information via the internet could potentially aid PCF education and impact on self efficacy of $\mathrm{CHI}$ management.

\section{Research questions}

1) Can video animations improve understanding of $\mathrm{CHI}$ among parents, carers and families of patients with $\mathrm{CHI}$ ?

2) Is video animation an acceptable method of information delivery among families and health care providers?

\section{Methods}

Following PCF consultation, three whiteboard video animations on aspects of $\mathrm{CHI}$ were produced using VideoScribe software (Sparkol, Clevedon, UK). After watching the videos, participants completed an anonymous web-based questionnaire. Respondents were grouped according to whether they were health care providers (HCPs, $n=8)$ or patient carers and families (PCFs, $n=12$ ) and data were evaluated using descriptive statistics.

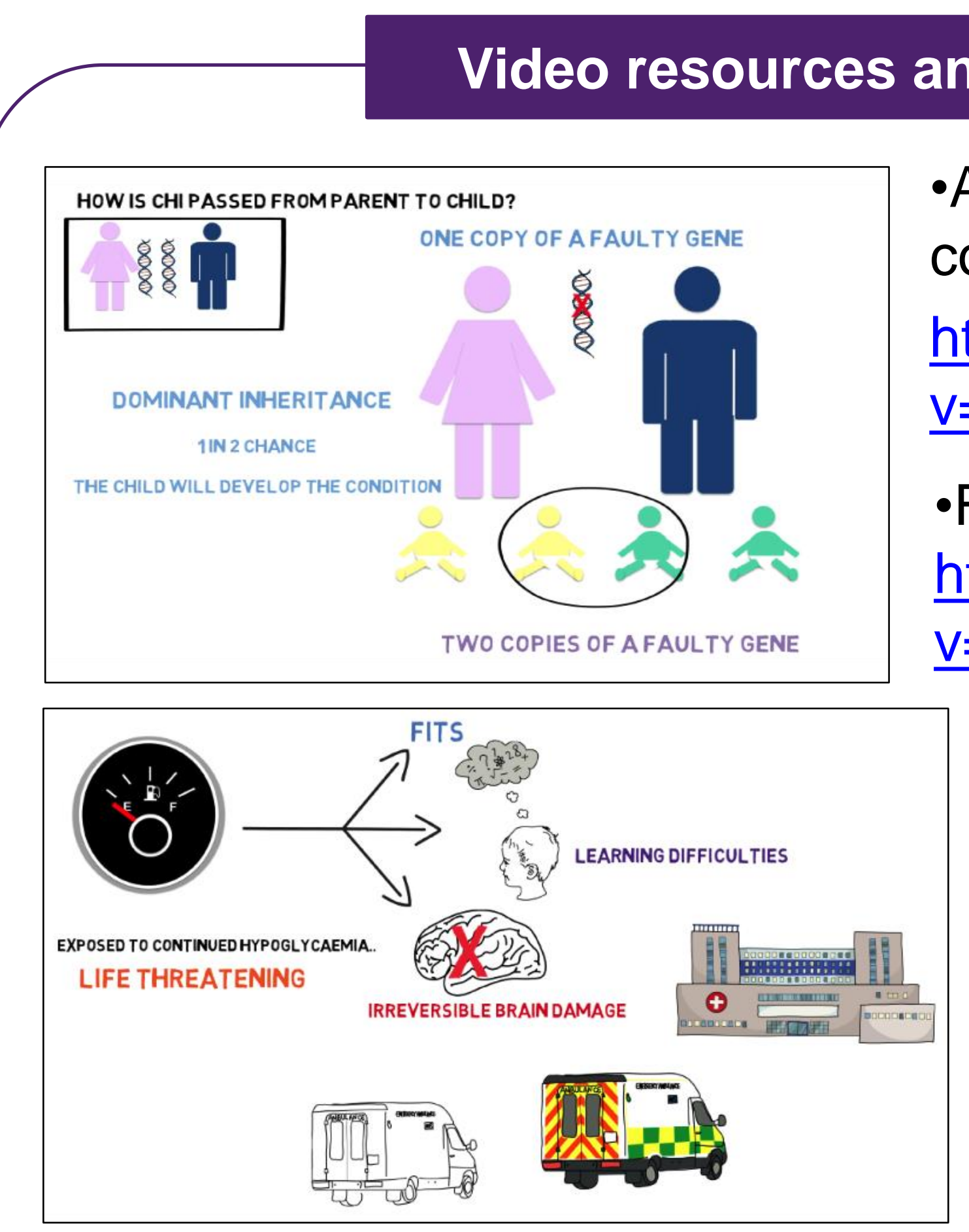

An introduction to the genetics of congenital hyperinsulinism

https://www.youtube.com/watch? $\mathrm{v}=\mathrm{qN} 3 \mathrm{Jd}$ WWOL-I

- Focal congenital hyperinsulinism https://www.youtube.com/watch? $\mathrm{v}=\mathrm{h}-5 \mathrm{XsG} 5 \mathrm{ICKQ}$

-The effects of hypoglycaemia in congenital hyperinsulinism https://www.youtube.com/watc $\underline{h ? v=r T 1 y 2 s C G T P C}$

Videos are now freely available via YouTube for use and distribution.

Results 1: Preferred sources of educational material

Biology knowledge was reported as below GCSE standard for $25 \%$ of PCFs. In seeking CHI-related information, $75 \%$ of PCFs used websites, $50 \%$ asked their HCP and $20 \%$ used social media. These data highlight the importance of simple, web-based materials for PCF education.

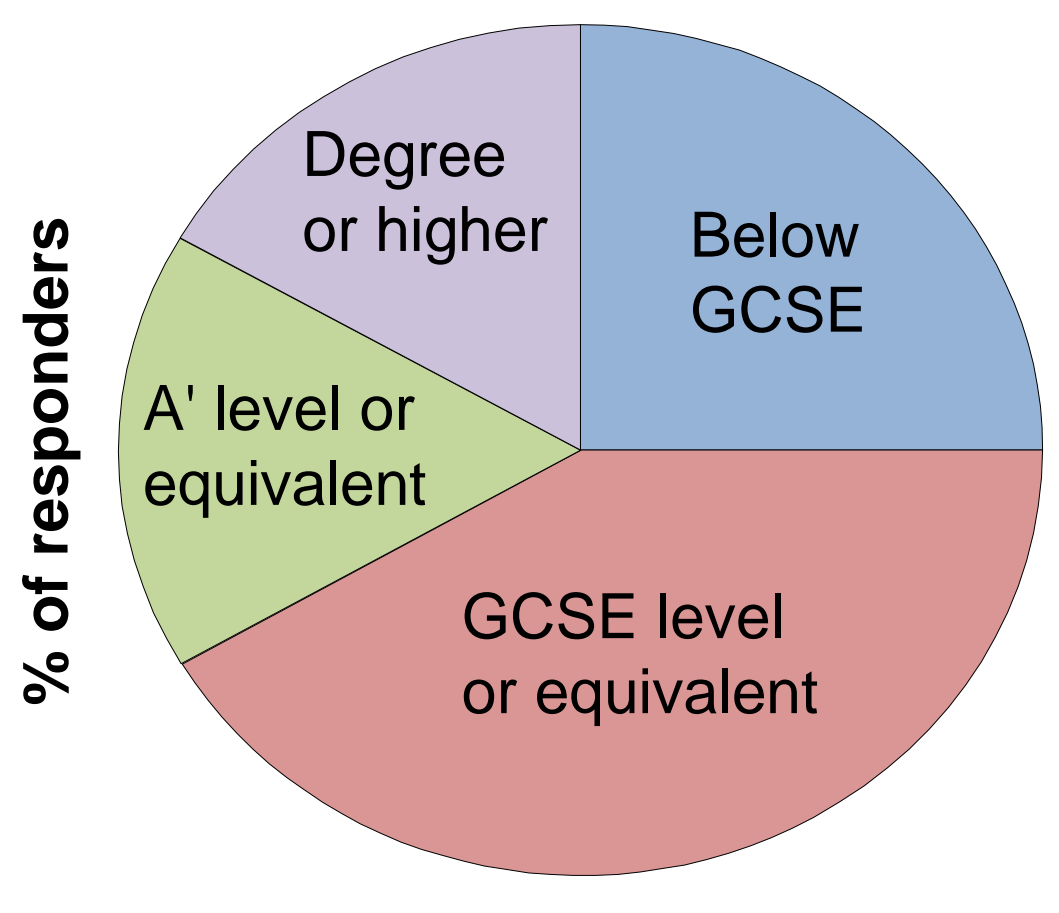

Fig. 1. The majority of parents carers, and families are educated in biology to at least GCSE level.

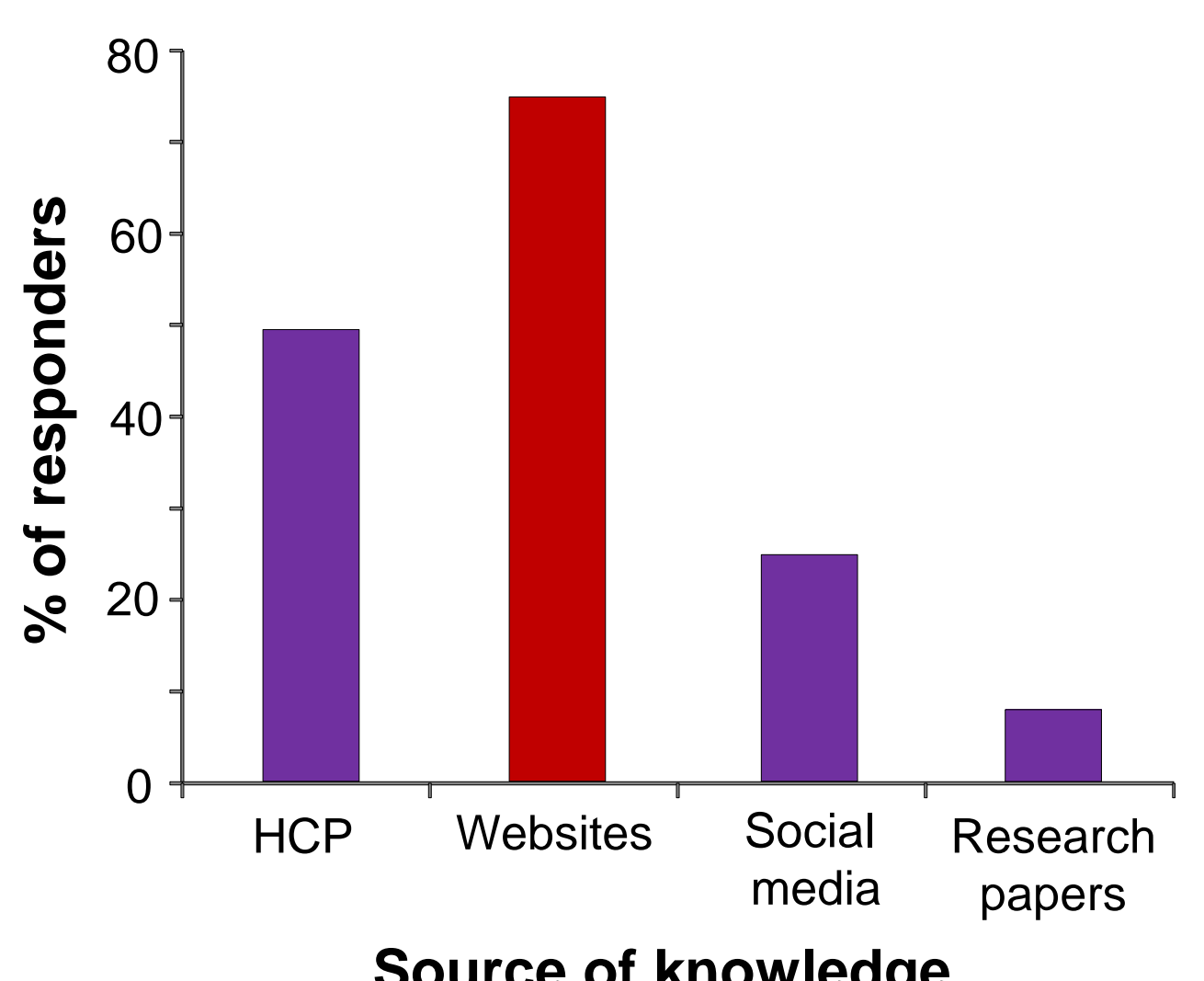

Source of knowledge

Fig. 2. Most parents, carers and families use websites for information on $\mathrm{CHI}$.
Results 2: Impact of videos

After watching the videos, $73 \%$ of PCFs reported improved understanding of $\mathrm{CHI}$ and $82 \%$ of PCFs felt that the videos improved their confidence to explain $\mathrm{CHI}$ to others. Overall $100 \%$ of respondents would recommend the video animations to friends, relatives, or other HCPs.

\section{Prior knowledge}

or experience
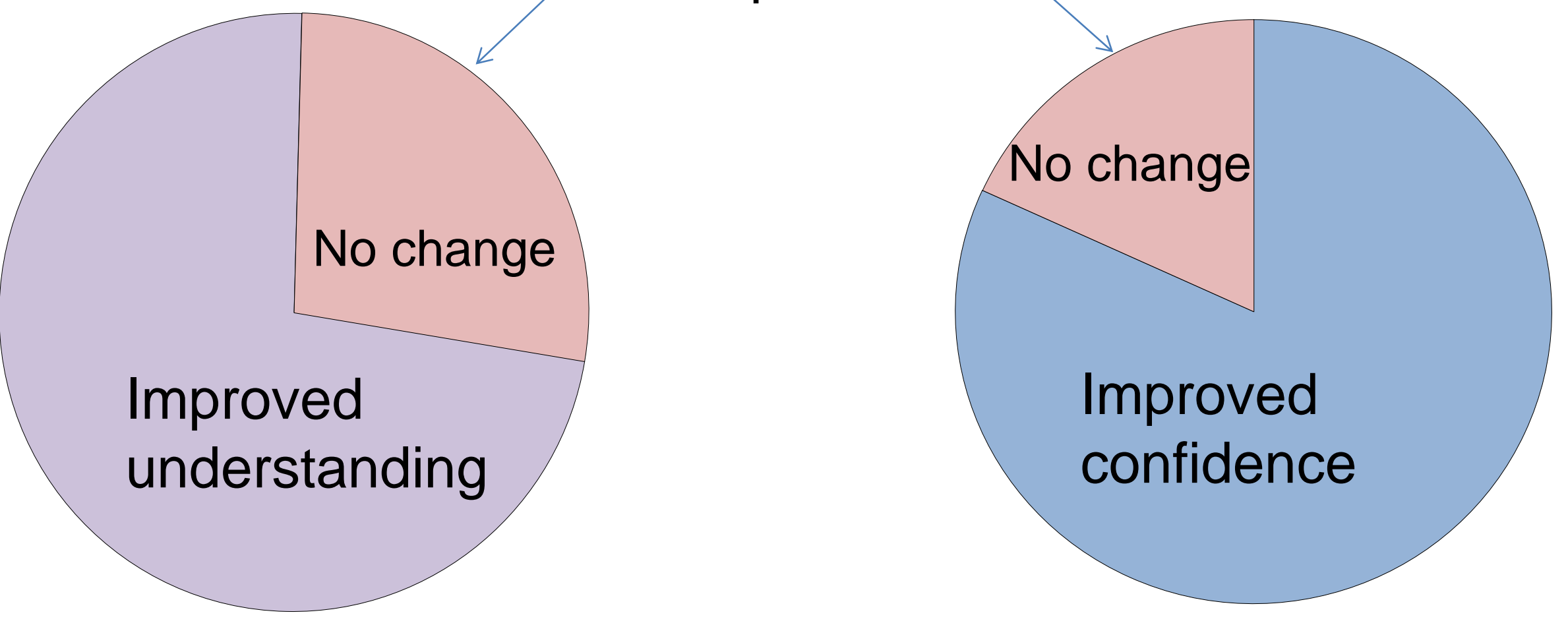

Fig. 3. The videos led to improved PCF self-reported understanding of $\mathrm{CHI}$.

Fig. 4. The videos led to improved PCF confidence to explain $\mathrm{CHI}$ to others.

\section{Results 3: Feedback from families}

Participants commented on both the positive aspects of the videos and aspects requiring improvement. A qualitative analysis revealed that the majority of respondents $(n=19)$ enjoyed the animated graphics.
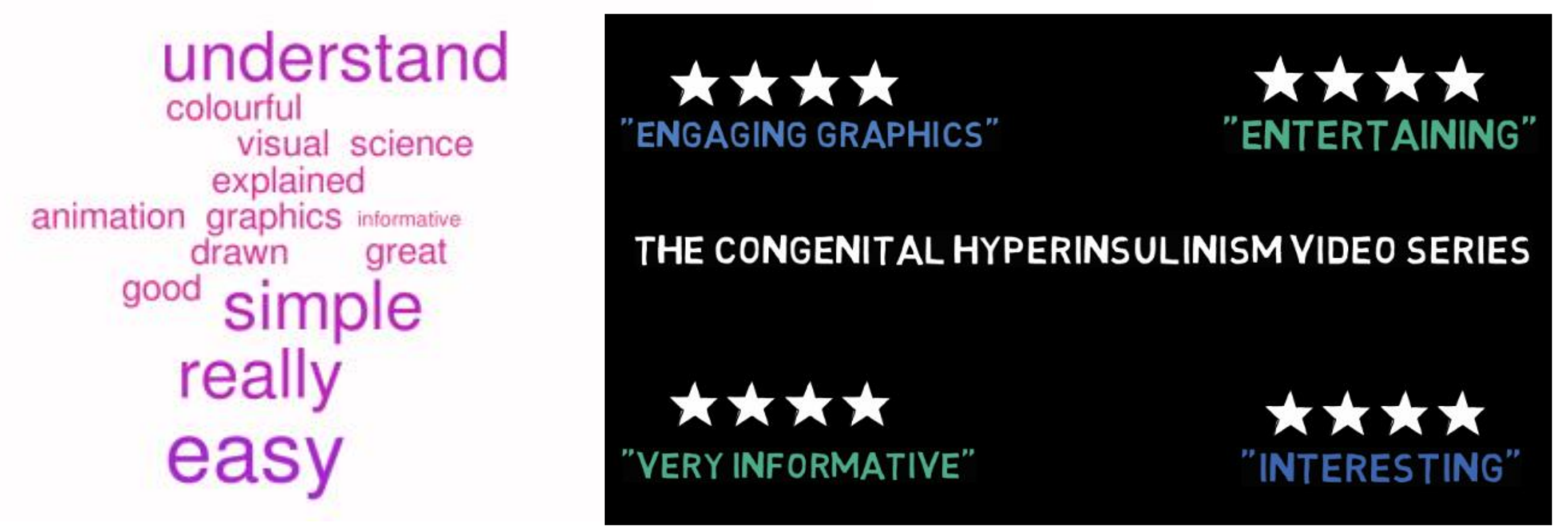

Areas identified as needing improvement included the speed of the videos, with $26 \%$ of respondents mentioning the videos could benefit from being slowed down. The original videos have now been rerecorded to take account of the suggestions made.

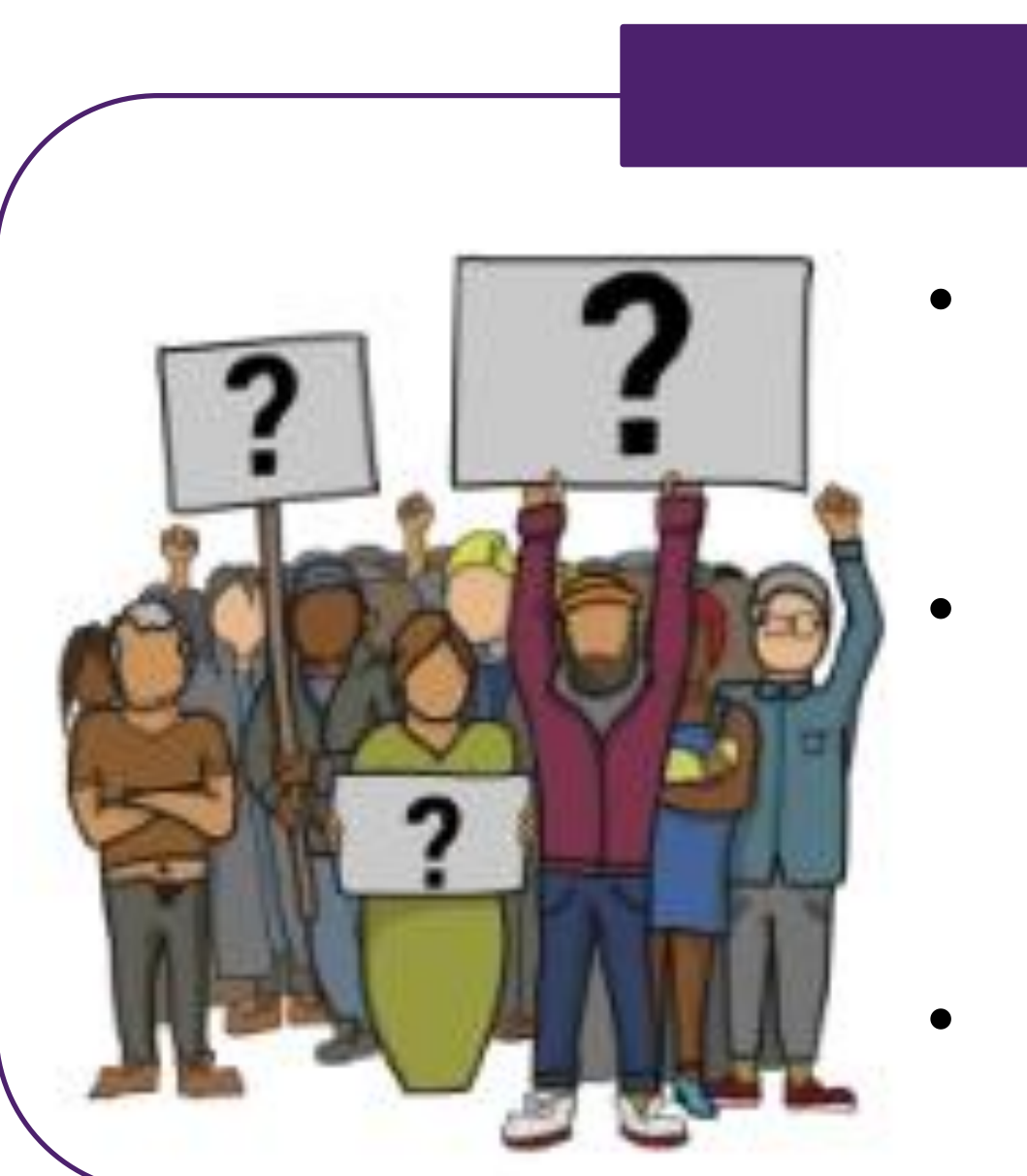

\section{Discussion}

The majority of PCFs use websites for further information about $\mathrm{CHI}$.

White-board video animation is simple to produce and effective at communicating challenging concepts to PCFs.

Video animation may aid retention and recall of information.

\section{Conclusions}

Our results suggest that animated videos communicate complex scientific concepts to patient families very effectively. Future work will attempt to measure the impact of these videos on family and patient outcome measures.

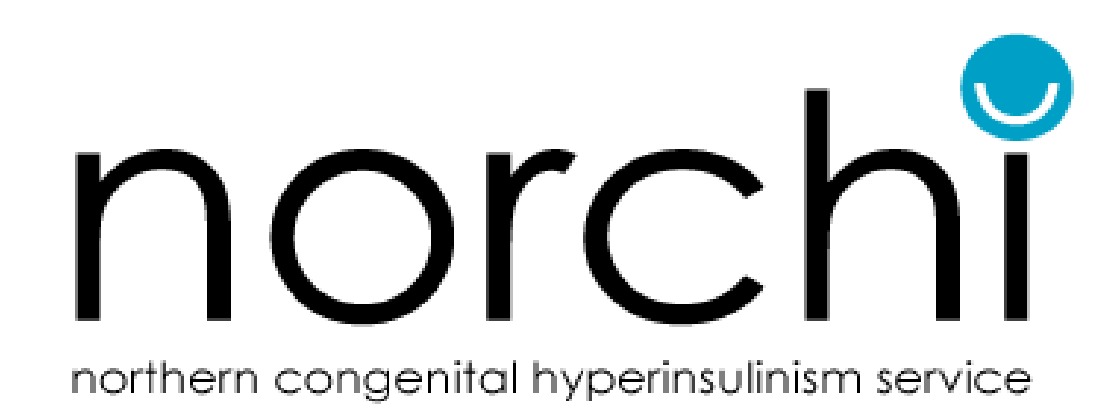

